Pacific Journal of Mathematics

TANGENT FRAME FIELDS ON SPIN MANIFOLDS 


\section{TANGENT FRAME FIELDS ON SPIN MANIFOLDS}

\section{DUANE RANDALL}

\section{In this note we prove the following theorems.}

Theorem A. Let $M^{n}$ be a spin manifold with $n \equiv 7 \bmod 8$ and $n>7$. Then $M$ admits at least 8 nonhomotopic tangent 4-frame fields.

Theorem B. Let $M^{n}$ be a spin manifold with $n \equiv 3 \bmod 8$ and $n>3$. Suppose that $w_{n-4} M=0$ and $w_{4} M \cdot w_{n-5} M=0$. Then $M^{n}$ admits a tangent 4 -frame field iff

$$
w_{n-3} M=0 \text { and } \chi_{2} M=0 \text {. }
$$

1. Introduction. Here $M^{n}$ denotes a closed connected smooth manifold of dimension $n$. A tangent $k$-frame field on $M^{n}$ is an ordered set of $k$ linearly independent vector fields on $M^{n}$. The classical theorem of Hopf states that $M^{n}$ possesses a tangent 1-frame field iff the Euler characteristic $\chi M=0$. A table of necessary and sufficient conditions for tangent 2-frame fields on orientable manifolds appears in [10] while conditions for tangent 3 -frame fields are tabulated in and [3]. In particular, Atiyah and Dupont prove in [1] that any orientable manifold $M^{n}$ with $n \equiv 3 \bmod 4$ admits a tangent 3 -frame field. This result is best possible since neither the sphere $S^{8 i+3}$ nor $S^{3} \times C P^{4 i+2}$ admits a tangent 4 -frame field.

Recall that an orientable manifold $M^{n}$ is called a spin manifold if the Stiefel-Whitney class $w_{2} M$ is trivial. The mod 2 semicharacteristic $\chi_{2} M^{n}$ is defined if $n=2 s+1$ by

$$
\chi_{2} M=\left(\sum_{i=0}^{s} \operatorname{dim} H_{i}(M ; Z / 2)\right) \bmod 2 .
$$

Let $\sigma M$ denote the signature of $M^{n}$ whenever $n$ is divisible by 4 . Finally $\delta$ represents the Bockstein-coboundary operator associated to the exact coefficient sequence $Z \rightarrow Z \rightarrow Z / 2$.

Theorem $\mathrm{A}$ is a best possible result for $n \equiv 7 \bmod 16$. In $[8, \mathrm{p}$. 690] Szczarba constructed certain spin manifolds $M^{n}$ with $n \equiv 3 \bmod 4$ as the quotient spaces of free and differentiable actions of generalized quarternion groups on $S^{n}$. The span of these spherical space forms $M^{n}$ with $n \equiv 7 \bmod 16$ and $n>7$ is precisely 4 by Theorem 1.1 of [2].

An immediate consequence of Theorem $\mathrm{A}$ and the result of Thurston given by [14, Corollary 1] is the following.

CoROLlaRY. Let $M^{n}$ be a spin manifold with $n \equiv 7 \bmod 8$ and 
$n>7$. Then $M$ possesses a $C^{\infty}$ codimension 4 foliation with trivial normal bundle.

We shall derive the following consequence of Theorem $\mathrm{A}$ and a theorem of Atiyah-Dupont given in [1, p. 25].

Proposition. Let $M^{n}$ be a spin manifold with $n \equiv 0 \bmod 8$ and $n>8$. Suppose that $H_{1}(M ; Z)$ has no 2-torsion, $\delta w_{n-6} M=0$, and $u^{2}=0$ for all $u$ in $H^{2}(M ; Z / 2)$. Then $M$ admits a tangent 5 -frame field iff

$$
w_{n-4} M=0, \chi M=0, \text { and } \sigma M \equiv 0 \bmod 16 .
$$

The above proposition was proved by Atiyah-Dupont under the assumption that $M^{n}$ is 3-connected. Both Theorem A and B were announced in [7] and generalize Theorem 1.2 of [9]. Indeed, their proofs are applications of the Postnikov methods developed by Emery Thomas and applied in [9], [11], [12], [13], [5], and [6]. We thank Samuel Gitler, James Heitsch, and Joāo de Carvalho for helpful conversations.

2. Proof of Theorem A. The $k$-invariants in a modified Postnikov resolution for the fibration

$$
V_{n, 4} \longrightarrow B \operatorname{Spin}(n-4) \stackrel{\pi}{\longrightarrow} B \operatorname{Spin}(n)
$$

through dimension $n$ where $n \equiv 3 \bmod 4$ and $n>7$ are listed with their defining relations below.

$$
\begin{aligned}
& k^{0}=w_{n-3} \\
& k^{1}: \mathrm{Sq}^{2} \mathrm{Sq}^{1} w_{n-3}=0 \\
& k^{2}:\left(\mathrm{Sq}^{4}+\cdot w_{4}\right) w_{n-3}=0 \\
& k^{3}: \mathrm{Sq}^{2} k^{1}=0
\end{aligned}
$$

(See resolution II of $[6$, p. 56].) Let

$$
\tau: M^{n} \longrightarrow B \operatorname{Spin}(n)
$$

classify the tangent bundle of $M$ where $n \equiv 7 \bmod 8$ and $n>7$. We must show that $\tau$ lifts to $B$ Spin $(n-4)$ in (2.1). Set $n=8 t+7$. Since the $W u$ classes $v_{i} M$ are trivial for $i>4 t$, the classes $w_{i} M$ are trivial for $i>8 t$ by the formula

$$
W=\operatorname{Sq} V .
$$

The proof of Theorem 1.3 of [11] evaluates $k^{1}(\tau)$ and $k^{3}(\tau)$ by secondary and tertiary operations applied to $w_{8 t+2} M=0$ respectively. Thus

$$
k^{1}(\tau)=0=k^{3}(\tau)
$$


because of zero indeterminacy. Let $U$ denote the Thom class of the Thom complex $T \tau$ associated to the tangent bundle $\tau$. In [9] Thomas proves that

$$
U \cdot k^{2}(\tau)=\psi(U)
$$

with zero indeterminacy where $\psi$ is a stable secondary operation associated to the relation in the Steenrod algebra

$$
\mathrm{Sq}^{4} \mathbf{S q}^{8 t+4}+\mathbf{S q}^{2}\left(\mathbf{S q}^{8 t+4} \mathbf{S q}^{2}\right)+\mathbf{S q}^{1}\left(\mathbf{S q}^{8 t+4} \mathbf{S q}^{3}+\mathbf{S q}^{8 t+6} \mathbf{S q}^{1}\right)=0 \text {. }
$$

We recall the following facts from [9] and [13]. Let

$$
s: M \times M \longrightarrow M \times M
$$

denote the involution which interchanges factors and let

$$
c: M \times M \longrightarrow T \tau
$$

denote the collapsing map associated to an embedding of $\tau$ as a neighborhood of the diagonal in $M \times M$. Select a basis

$$
\alpha_{1}, \cdots, \alpha_{r}
$$

for the graded vector space $\sum_{i=0}^{[n / 2]} H^{i}(M ; Z / 2)$. Let $\beta_{1}, \cdots, \beta_{r}$ be the dual basis by Poincaré duality such that

$$
\alpha_{i} \cdot \beta_{j}=\delta_{i j} \mu
$$

if $\operatorname{deg} \alpha_{i}+\operatorname{deg} \beta_{j}=n$. Here $\mu$ generates $H^{n}(M ; Z / 2)$ while clearly $r=\chi_{2} M$. We set

$$
A=\sum_{i=1}^{r} \alpha_{i} \otimes \beta_{i} .
$$

Then $c^{*} U=A+s^{*} A$ and $A \cdot s^{*} A=\chi_{2} M(\mu \otimes \mu)$.

Suppose that $\psi(A)$ is defined. The indeterminacy of $\psi(A)$ is trivial iff $w_{4} M=0$ since

$$
\mathrm{Sq}^{4}(v \otimes \mu)=\mathrm{Sq}^{4} v \otimes \mu=v \cdot w_{4} M \otimes \mu
$$

for any class $v$ in $H^{n-4}(M ; Z / 2)$. We consider the universal example $(E, m, v)$ for the operation $\psi$ on classes of dimension $8 t+7$.

$$
\Omega C \stackrel{i}{\longrightarrow} E \stackrel{p}{\longrightarrow} K(Z / 2,8 t+7) .
$$

Here $p$ is the principal fibration induced from the path-loop fibration on

$$
C=K(Z / 2,16 t+11) \times K(Z / 2,16 t+13) \times K(Z / 2,16 t+14)
$$

by the classifying map $K(Z / 2,8 t+7) \rightarrow C$ with component operations 


$$
\left(\mathrm{Sq}^{8 t+4}, \mathrm{Sq}^{8 t+4} \mathrm{Sq}^{2}, \mathrm{Sq}^{8 t+4} \mathrm{Sq}^{3}+\mathrm{Sq}^{8 t+6} \mathrm{Sq}^{1}\right)
$$

applied to the fundamental class $\iota$ of $K(Z / 2,8 t+7)$. Now $m$ denotes the homotopy-commutative multiplication on $E$ while $v$ in $H^{2 n}(E ; Z / 2)$ represents $\psi$.

We now exploit a technique of [4] in order to evaluate $\psi(U)$.

Let

$$
\bar{A}: M \times M \longrightarrow E
$$

denote any lifting of the class $A$ in (2.4) under the assumption that $\psi(A)$ is defined. Then the map (2.6) $g=m \circ(\bar{A}, \bar{A} \circ s): M \times M \rightarrow E$ defines a lifting of $A+s^{*} A$ such that

$$
g^{*} v=\bar{A}^{*} v+s^{*} \bar{A} v=0
$$

since $s^{*}$ is the identity on $H^{2 n}(M \times M ; Z / 2)$.

Let

$$
\bar{U}: T \tau \longrightarrow E
$$

be any lifting of the Thom class $U$ and set $f=\bar{U}_{\circ} c$. Since $c^{*}$ is a monomorphism, $\psi(U)$ vanishes if we can show that

$$
c^{*} \psi(U)=f^{*} v=g^{*} v=0 .
$$

Since $f$ and $g$ are liftings of $c^{*} U$, there exists a map

$$
h: M \times M \longrightarrow \Omega C
$$

unique up to homotopy such that $f$ and $m(i \circ h, g)$ are homotopic. We identify $h$ with a triple $(x, y, z)$ of classes in $H^{*}(M \times M ; Z / 2)$. Thus

$$
f^{*} v=g^{*} v+\mathrm{Sq}^{4} x+\mathrm{Sq}^{2} y+\mathrm{Sq}^{1} z=\mathrm{Sq}^{4} x \text {. }
$$

The map $i \circ h$ is invariant under $s$ since both $f$ and $g$ are invariant. Thus the homotopy class $[h]+[h \circ s]$ lies in the image of

$$
[M \times M, K(Z / 2,8 t+6)] \longrightarrow[M \times M, \Omega C] .
$$

Consequently,

$$
x+s^{*} x \varepsilon \mathrm{Sq}^{8 t+4} H^{8 t+6}(M \times M ; Z / 2) .
$$

Note that $\mathrm{Sq}^{4}$ is trivial on any class in $H^{i}(M ; Z / 2) \otimes H^{2 n-4-i}(M ; Z / 2)$ with bi-degree $(i, 2 n-4-i)$ different from $(n-4, n)$ and $(n, n-4)$.

The following lemma implies by (2.8) that the symmetric class $x+s^{*} x$ contains no nontrivial classes of bi-degree $(n-4, n)$ or $(n, n-4)$. Thus $x$ is symmetric in the classes with bi-degree $(n, n-4)$ and $(n-4, n)$. We conclude that 


$$
0=\mathrm{Sq}^{4} x=f^{*} v
$$

LEMMA. Let $M^{n}$ be any orientable manifold with $n=4 j+3$ and $j>0$.

Let

$$
P: H^{2 n-4}(M \times M ; Z / 2) \longrightarrow H^{n-4}(M ; Z / 2) \otimes H^{n}(M ; Z / 2)
$$

be the projection morphism corresponding to the Kunneth formula. Then the kernel of $P$ contains

$$
\mathrm{Sq}^{n-3} H^{n-1}(M \times M ; Z / 2) .
$$

Proof. Let $\alpha \otimes \beta$ be a class with bi-degree $(i, 4 j+2-i)$ in $H^{n-1}(M \times M ; Z / 2)$. By the Cartan formula and dimensionality

$$
\mathrm{Sq}^{4 j}(\alpha \otimes \beta)=\alpha^{2} \otimes \mathrm{Sq}^{4 j-2} \beta+\mathrm{Sq}^{i-1} \alpha \otimes \mathrm{Sq}^{4 j-i+1} \beta+\mathrm{Sq}^{i-2} \alpha \otimes \mathrm{Sq}^{4 j-i+2} \beta \text {. }
$$

The image of $\operatorname{Sq}^{4 j}(\alpha \otimes \beta)$ under $P$ is clearly trivial unless $i=2 j$. Further,

$$
\begin{aligned}
& \mathrm{Sq}^{2 j+1} \beta=\mathrm{Sq}^{1} \mathrm{Sq}^{2 j} \beta=0 \text { in } \\
& H^{n}(M ; Z / 2) \text { when } i=2 j .
\end{aligned}
$$

To complete the proof of Theorem A, we must justify the assumption that $\psi(A)$ is defined. We leave this verification to the reader, since we shall make similar calculations in the more complicated proof of Theorem B. Finally, by [1, Proposition 6.13], the existence of a tangent 4 -frame field on $M$ given by a lifting of $\tau$ to $B$ Spin $(n-4)$ implies the existence of 8 nonhomotopic tangent 4 -frame fields.

REMARK. The proof of Theorem A shows that any lifting of $\tau$ to any stage in the Postnikov resolution itself lifts to $B$ Spin $(n-4)$ since all the $k$-invariants of $\tau$ are trivial with zero indeterminacy.

3. Proof of Theorem B. Let $M^{n}$ be a spin manifold with $n=$ $8 t+3$ for positive $t$ such that

$$
w_{4} M \cdot w_{n-5} M=0 \text { and } w_{n-4} M=0 .
$$

We adopt the notation of $\S 2$ freely. We must show that

$$
\tau: M \longrightarrow B \operatorname{Spin}(n)
$$

has a lifting in the fibration (2.1) iff

$$
w_{n-3} M=0 \text { and } \chi_{2} M=0 .
$$

Suppose the primary obstruction $w_{8 t} M$ vanishes. For $n=11$, 
the obstructions $k^{1}(\tau)$ and $k^{3}(\tau)$ vanish since they lie in the image of $\mathrm{Sq}^{2}$ and a spin-trivial secondary operation respectively. For $n>11$, the proof of Theorem 1.3 of [11] establishes the triviality of $k^{1}(\tau)$ and $k^{3}(\tau)$, whenever defined. (Note corrigenda (ii) in [10].)

In [9] Thomas proves that

$$
U \cdot k^{2}(\tau)=\Gamma(U)
$$

with zero indeterminacy where $\Gamma$ is a nonstable secondary operation associated to the relation

$$
\mathrm{Sq}^{4} \mathrm{Sq}^{8 t}+\mathrm{Sq}^{1}\left(\mathrm{Sq}^{8 t+2} \mathbf{S q}^{1}+\mathbf{S q}^{8 t} \mathbf{S q}^{3}\right)+\mathbf{S q}^{2}\left(\mathbf{S q}^{8 t} \mathbf{S q}^{2}\right)=0
$$

which holds on mod 2 classes of degree $<8 t+4$. Let $(E, m, v)$ denote the universal example for the operation $\Gamma$ on classes of degree $8 t+3$. Since $\Gamma$ is nonstable,

$$
m^{*} v=v \otimes 1+1 \otimes v+p^{*} \iota \otimes p^{*} \iota
$$

in $H^{2 n}(E \times E ; Z / 2)$. Suppose $\Gamma(A)$ is defined. The map $g$ in (2.6) associated to any lifting $\bar{A}$ defines a lifting of $c^{*} U$ such that

$$
g^{*} v=\bar{A}^{*} v+s^{*} \bar{A}^{*} v+A \cdot s^{*} A=\chi_{2} M(\mu \otimes \mu) .
$$

Let $\bar{U}: T \tau \rightarrow E$ be any lifting of the Thom class $U$ and set $f=$ $\bar{U} \circ c$. The argument in $\S 2$ shows that

$$
f^{*} v=g^{*} v \text {. }
$$

(Recall that the lemma in $\S 2$ was formulated for $n \equiv 3 \bmod 4$.) Thus

$$
U \circ k^{2}(\tau)=\Gamma(U)=\bar{U}^{*} v=\left(\chi_{2} M\right) U \cdot \mu
$$

and so by the Thom isomorphism

$$
k^{2}(\tau)=\left(\chi_{2} M\right) \mu .
$$

The following lemma concludes the proof of Theorem B.

Lemma. $\quad \Gamma(A)$ is defined.

Proof. Now $\mathrm{Sq}^{8 t+2} \mathrm{Sq}^{1} A=0=\mathrm{Sq}^{8 t} \mathrm{Sq}^{3} A$ in the spin manifold $M \times M$ since

$$
\begin{aligned}
& \mathrm{Sq}^{8 t+2} \mathrm{Sq}^{1}=\mathrm{Sq}^{2}\left(\mathrm{Sq}^{8 t} \mathbf{S q}^{1}\right) \\
& \mathrm{Sq}^{8 t} \mathrm{Sq}^{3}=\mathrm{Sq}^{2}\left(\mathrm{Sq}^{8 t-1} \mathbf{S q}^{2}\right)+\mathrm{Sq}^{1}\left(\mathrm{Sq}^{8 t} \mathrm{Sq}^{2}\right)
\end{aligned}
$$

Note that $\mathrm{Sq}^{2} A$ is symmetric since

$$
\mathrm{Sq}^{2} A+s^{*} \mathrm{Sq}^{2} A=c^{*} \mathrm{Sq}^{2} U=0 \text {. }
$$


Thus $\mathrm{Sq}^{2} A$ contains nonzero summands only of bi-degree $(4 t+2,4 t+3)$ and $(4 t+3,4 t+2)$. Let $\beta \otimes \gamma$ be any class with bi-degree $(4 t+1$, $4 t+2)$. Now

$$
\mathrm{Sq}^{4 t} \mathrm{Sq}^{1} \gamma=\mathrm{Sq}^{2} \mathrm{Sq}^{4 t-1} \gamma+\mathrm{Sq}^{1} \mathrm{Sq}^{4 t} \gamma=0
$$

so by the Cartan formula

$$
\mathrm{Sq}^{8 t} \mathrm{Sq}^{2} A=\sum \mathrm{Sq}^{4 t} \mathrm{Sq}^{2} \alpha_{i} \otimes \mathrm{Sq}^{4 t} \beta_{i}
$$

where only the summands with degree $\alpha_{i}=4 t$ or $4 t+1$ are possibly nonzero.

Suppose that the Wu class $v_{4 t}=0$. Then

$$
\mathrm{Sq}^{4 t} \beta=\beta \cdot v_{4 t}=0
$$

for any $\beta$ in $H^{4 t+3}(M ; Z / 2)$. If $v_{4 t}$ is nonzero, we are free to choose $v_{4 t}$ to be a class in (2.3). Set $\alpha_{j}=v_{4 t}$. We consider any summand in (3.1) with

$$
\text { degree } \alpha_{i}=4 t \text {, degree } \beta_{i}=4 t+3 \text {. }
$$

Now $\mathrm{Sq}^{4 t} \beta_{i}=\beta_{i} \cdot v_{4 t}=\beta_{i} \cdot \alpha_{j}=0$ for $i \neq j$. If $i=j$,

$$
\mathrm{Sq}^{4 t} \mathrm{Sq}^{2} \alpha_{j}=\mathrm{Sq}^{4} \mathrm{Sq}^{4 t-2} v_{4 t} \text {. }
$$

By dimensionality $\mathrm{Sq}^{4 t-2} v_{4 t}=w_{8 t-2} M$. We conclude that

$$
\mathrm{Sq}^{4 t} \mathrm{Sq}^{2} \alpha_{j}=\mathrm{Sq}^{4} w_{n-5} M=w_{4} M \cdot w_{n-5} M=0 .
$$

But all summands in (3.1) with degree $\alpha_{i}=4 t+1$ must vanish by symmetry so

$$
\mathrm{Sq}^{8 t} \mathrm{Sq}^{2} A=0 \text {. }
$$

The class $\mathrm{Sq}^{8 t} A$ is symmetric since

$$
\mathrm{Sq}^{8 t} A+s^{*} \mathrm{Sq}^{8 t} A=c^{*} \mathrm{Sq}^{8 t} U=0 .
$$

Recall that degree $\alpha_{i} \leqq 4 t+1$ for every $\alpha_{i}$ in (2.3). By symmetry the possibly nonzero summands in $\mathrm{Sq}^{8 t} A$ are the classes

$$
\mathrm{Sq}^{4 t+1} \alpha_{i} \otimes \mathrm{Sq}^{4 t-1} \beta_{i}+\mathrm{Sq}^{4 t} \alpha_{i} \otimes \mathrm{Sq}^{4 t} \beta_{i}
$$

where $\alpha_{i} \otimes \beta_{i}$ has bi-degree $(4 t+1,4 t+2)$.

We claim that either $\mathrm{Sq}^{4 t} \alpha_{i}$ or $\mathrm{Sq}^{4 t} \beta_{i}$ is trivial. Choose a basis

$$
x_{1} v_{4 t}, x_{2} v_{4 t}, \cdots, x_{j} v_{4 t}
$$

for $v_{4 t} H^{1}(M ; Z / 2)$. Extend this basis to a basis

$$
\alpha_{1}, \cdots, \alpha_{r}
$$


for $H^{4 t+1}(M ; Z / 2)$ with $\alpha_{i}=x_{i} v_{4 t}$ for $i \leqq j$. Let $\beta_{1}, \cdots, \beta_{r}$ denote the dual basis for $H^{4 t+2}(M ; Z / 2)$. For $j<i \leqq r$ and any class $z$ in $H^{1}(M ; Z / 2)$,

$$
\mathrm{Sq}^{4 t} \beta_{i} \cdot z=\mathrm{Sq}^{4 t}\left(\beta_{i} z\right)=\beta_{i}\left(z v_{4 t}\right)=0 .
$$

Thus $\mathrm{Sq}^{4 t} \beta_{i}=0$ for $j<i$. For $i \leqq j$

$$
\mathrm{Sq}^{4 t}\left(x_{i} v_{4 t}\right)=x_{i} w_{n-3} M+x_{i}^{2} w_{n-4} M=0 \text {. }
$$

We conclude by symmetry that $\mathrm{Sq}^{8 t} A=0$.

4. Proof of Proposition. Let $M^{n}$ be a spin manifold with $n \equiv 0 \bmod 8$ and $n>8$. We assume that $H_{1}(M ; Z)$ has no 2 -torsion, $\delta w_{n-6} M=0$, and $u^{2}=0$ for all $u$ in $H^{2}(M ; Z / 2)$. Let

$$
\tau: M \longrightarrow B \operatorname{Spin}(n)
$$

classify the tangent bundle of $M$. The following diagram is the Moore-Postnikov resolution for the fibration

$$
\pi: B \operatorname{Spin}(n-5) \longrightarrow B \operatorname{Spin}(n)
$$

through dimension $n$.

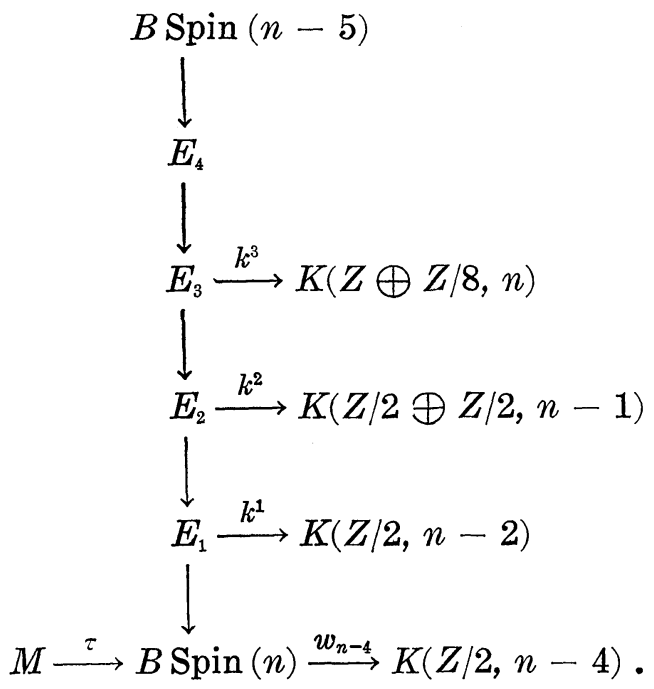

Let $f: M \longrightarrow E_{3}$ be any lifting for $\tau$. Then

$$
f^{*} k^{3} \in H^{n}(M ; Z \oplus Z / 8) \cong Z \oplus Z / 8 \text {. }
$$

Atiyah and Dupont in [1, p. 25] show that

$$
f^{*} k^{3}=(0,0) \text { iff } \chi M=0 \text { and } \sigma M \equiv 0 \bmod 16 .
$$


We must show that $\tau$ lifts to $E_{3}$ iff $w_{n-4} M=0$. Assume $w_{n-4} M=0$ so $\tau$ lifts to $E_{1}$.

The following diagram contains the first stage of a modified Postnikov resolution for the fibration

$$
B \operatorname{Spin}(n-6) \longrightarrow B \operatorname{Spin}(n)
$$

through dimension $n-1$.

$$
\begin{aligned}
& \bar{E}_{1}
\end{aligned}
$$

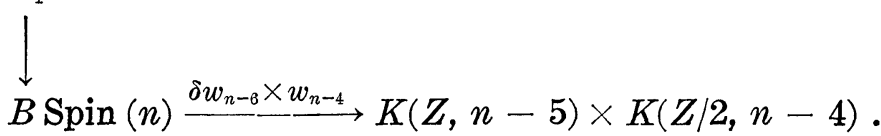

Let $h: \bar{E}_{1} \rightarrow E_{1}$ denote the induced map.

Then

$$
h^{*} k^{1}=\mathrm{Sq}^{2} y
$$

where $y$ has the defining relation

$$
\mathrm{Sq}^{2}\left(\delta w_{n-6}\right)+\mathrm{Sq}^{1} w_{n-4}=0 \text {. }
$$

The map $\tau$ lifts to $\bar{E}_{1}$ since $\delta w_{n-6} M=0=w_{n-4} M$. The indeterminacy of $k^{1}(\tau)$ is given by

$$
\mathrm{Sq}^{2} \mathrm{Sq}^{1} H^{n-5}(M ; Z / 2)=0 \text {. }
$$

Now $\mathrm{Sq}^{2}$ vanishes on $H^{n-4}(M ; Z / 2)$ iff $u^{2}=0$ for all $u$ in $H^{2}(M ; Z / 2)$ by Poincaré duality and the Cartan formula. We conclude that $\tau\left(k^{1}\right)=0$ so $\tau$ lifts to $E_{2}$ in (4.1).

We write $g^{*} k^{2}=(u, v)$ where $g: M \rightarrow E_{2}$ is any lifting of $\tau$ and the classes $u$ and $v$ belong to $H^{n-1}(M ; Z / 2)$. Suppose that $g^{*} k^{2}$ is nonzero. Then at least one class, say $u$, is nontrivial. Now

$$
0=\delta u \in H^{n}(M ; Z) \approx Z \text {. }
$$

Select any class $x$ in $H^{n-1}(M ; Z)$ such that $\rho_{2} x=u$ where $\rho_{2}$ denotes reduction mod 2. Next choose a class $a$ in $H_{n-1}(M ; Z)$ such that the evaluation $x(\alpha)$ is an odd multiple of a generator for $H_{0}(M ; Z) \approx Z$. There exists such a class $a$ because $H^{n-1}(M ; Z)$ has no 2-torsion.

Let $i: N \rightarrow M$ be the inclusion of an oriented codimension one submanifold $N$ (not necessarily connected) of $M$ such that

$$
i_{*}\left(\mu_{N}\right)=a .
$$

Here $\mu_{N}$ denotes the fundamental homology class of $N$. Since

$$
x(\alpha)=x\left(i_{*} \mu_{N}\right)=\left(i^{*} x\right)\left(\mu_{N}\right),
$$


it follows that $i^{*} u=\rho_{2}\left(i^{*} x\right) \neq 0$. Note that the lifting

$$
g \circ i: N \longrightarrow E_{2}
$$

of the stable tangent bundle of $N$ does not lift to $E_{3}$ since

$$
(g \circ i)^{*} k^{2}=\left(i^{*} u, i^{*} v\right) \neq(0,0) .
$$

The following lemma applied to the connected components of $N$ yields a contradiction to the assumption that $g^{*} k^{2}$ is nonzero. Thus $\tau$ lifts to $E_{3}$ and the proposition is proved.

Lemma. Let $N$ be any codimension 1, closed, connected, orientable submanifold of $M$ with inclusion denoted by $i$. Then any lifting of

$$
\tau \circ i: N \longrightarrow B \operatorname{Spin}(n)
$$

to any space $E_{j}$ in the resolution (4.1) further lifts to $B$ Spin $(n-5)$.

Proof. The normal bundle to $N$ in $M$ is trivial by orientability. So $N$ is a spin manifold whose stable tangent bundle is classified by the composite $\tau \circ i$. The Moore-Postnikov resolution in (4.1) is essentially a modified Postnikov resolution through dimension $n-1$. One component of the class $k^{2}$ is the image of a class $z$ in $H^{n-1}\left(E_{1} ; Z / 2\right)$ with defining relation

$$
\left(\mathrm{Sq}^{4}+\cdot w_{4}\right) w_{n-4}=0 .
$$

The corresponding spaces in the modified Postnikov resolution (2.1) for the fibration

$$
B \operatorname{Spin}(n-5) \longrightarrow B \operatorname{Spin}(n-1)
$$

clearly map into $E_{1}$ and $E_{2}$ in (4.1). The map of resolutions begins with the inclusion

$$
B \operatorname{Spin}(n-1) \longrightarrow B \operatorname{Spin}(n) \text {. }
$$

With respect to the induced maps, the class $z$ goes to $k^{2}$ in (2.1) while the other component of $k^{2}$ in (4.1) maps to $k^{3}$ in (2.1). The proof of Theorem A shows that any lifting of $\tau(N)$ to any stage in the modified Postnikov resolution (2.1) for the fibration

$$
B \operatorname{Spin}(n-5) \longrightarrow B \operatorname{Spin}(n-1)
$$

itself lifts to $B$ Spin $(n-5)$. (See the remark in $\S 2$.) Thus the same property holds for any lifting of the stable tangent bundle in the resolution (4.1). 


\section{REFERENCES}

1. M. F. Atiyah and J. L. Dupont, Vector fields with finite singularities, Acta Math., 128 (1972), 1-40.

2. J. C. Becker, Vector fields on quotient manifolds of spheres, Indiana Univ. Math. J., 22 (1973), 859-871.

3. J. L. Dupont, K-theory obstructions to the existence of vector fields, Acta Math., 133 (1974), 67-80.

4. M. Mathowald, The index of a tangent 2-field, Pacific J. Math., 58 (1975), 539-548.

5. D. Randall, Some immersion theorems for projective spaces, Trans. Amer. Math. Soc., 147 (1970), 135-151.

6. - Some immersion theorems for manifolds, Trans. Amer. Math. Soc., 156 (1971), 45-58.

7. - Remarks on vector fields, To appear in Atas Brasil Math. Colloq. in Poços de Caldas, (1975).

8. R. H. Szczarba, On tangent bundles of fibre spaces and quotient spaces, Amer. J. Math., 86 (1964), 685-697.

9. E. Thomas, The span of a manifold, Quart. J. Math., 19 (1968), 225-244.

10. - Vector fields on manifolds, Bull. Amer. Math. Soc., 75 (1969), 643-683.

11. - Real and complex vector fields on manifolds, J. Math. Mech., 16 (1967), $1183-1206$.

12. - Postnikov invariants and higher order cohomology operations, Annals of Math., 85 (1967), 184-217.

13. - The index of a tangent 2-field, Comment. Math. Helv., 42 (1967), 86-110. 14. W. Thurston, The theory of foliations of codimension greater than one, Comment. Math. Helv., 49 (1974), 214-231.

Received August 1, 1977. This research was supported by funds from CNPq, CAPES, and FINEP of Brasil.

Pontifícia Universidade Católica do

RIO DE JANEIRO-BRASIL 



\section{PACIFIC JOURNAL OF MATHEMATICS}

\section{EDITORS}

RICHARD ARENS (Managing Editor)

University of California

Los Angeles, California 90024

C. W. CURTIS

University of Oregon

Eugene, OR 97403

C. C. MOORE

University of California

Berkeley, CA 94720

\section{J. DUGUNDJI}

Department of Mathematics University of Southern California Los Angeles, California 90007

R. Finn aNd J. Milgram Stanford University Stanford, California 94305

\section{ASSOCIATE EDITORS}

E. F. BeCKenbaCH

B. H. NeumanN

F. WOLF

K. YosHIDA

\section{SUPPORTING INSTITUTIONS}

UNIVERSITY OF BRITISH COLUMBIA CALIFORNIA INSTITUTE OF TECHNOLOGY UNIVERSITY OF CALIFORNIA MONTANA STATE UNIVERSITY UNIVERSITY OF NEVADA, RENO NEW MEXICO STATE UNIVERSITY OREGON STATE UNIVERSITY UNIVERSITY OF OREGON
UNIVERSITY OF SOUTHERN CALIFORNIA STANFORD UNIVERSITY UNIVERSITY OF HAWAII UNIVERSITY OF TOKYO UNIVERSITY OF UTAH WASHINGTON STATE UNIVERSITY UNIVERSITY OF WASHINGTON 


\section{Pacific Journal of Mathematics}

\section{Vol. 76, No. $1 \quad$ November, 1978}

Ata Nuri Al-Hussaini, Potential operators and equimeasurability ......... 1

Tim Anderson and Erwin Kleinfeld, Semisimple nil algebras of type $\delta . \ldots .99$

Stephen LaVern Campbell, Linear operators for which $T^{*} T$ and $T+T^{*}$

commute. III ......................................

Robert Jay Daverman, Special approximations to embeddings of codimension one spheres...............................

Donald M. Davis, Connective coverings of $\mathrm{BO}$ and immersions of projective

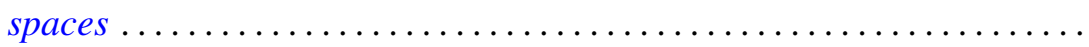

V. L. (Vagn Lundsgaard) Hansen, The homotopy type of the space of maps of

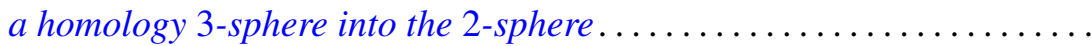

James Victor Herod, A product integral representation for the generalized

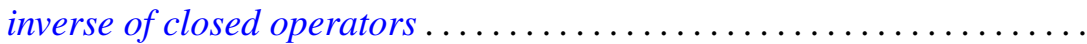

A. A. Iskander, Definability in the lattice of ring varieties ..............

Russell Allan Johnson, Existence of a strong lifting commuting with a

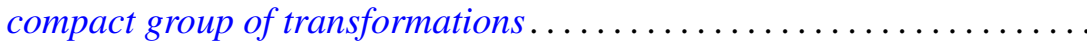

Heikki J. K. Junnila, Neighbornets...................... 83

Klaus Kalb, On the expansion in joint generalized eigenvectors . ......... 109

F. J. Martinelli, Construction of generalized normal numbers . . . . . . . . . 117

Edward O'Neill, On Massey products ....................... 123

Vern Ival Paulsen, Continuous canonical forms for matrices under unitary

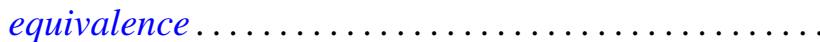

Justin Peters and Terje Sund, Automorphisms of locally compact groups . . . 143

Duane Randall, Tangent frame fields on spin manifolds . . . .

Jeffrey Brian Remmel, Realizing partial orderings by classes of co-simple sets . . . .

J. Hyam Rubinstein, One-sided Heegaard splittings of 3-manifolds ...

Donald Charles Rung, Meier type theorems for general boundary approach

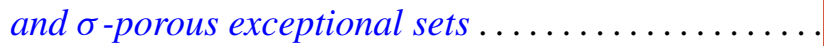

Ryōtarō Satō, Positive operators and the ergodic theorem

Ira H. Shavel, A class of algebraic surfaces of general type constructed from

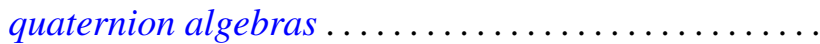

Patrick F. Smith, Decomposing modules into projectives and injectives ....

Sergio Eduardo Zarantonello, The sheaf of outer functions in the polydisc... 